\title{
Sensitivity analysis of truck structure based on CA algorithm
}

\author{
Xiaochen Yang ${ }^{1}$, Yuchen Jin $^{1}$, Rui Feng ${ }^{1}$ and Guikai Guo ${ }^{1 *}$ \\ ${ }^{1}$ School of Mechanical and Aerospace Engineering, Jilin University, Changchun, People's Republic of China
}

\begin{abstract}
With the continuous improvement of modern CAE technology, structural reanalysis algorithm has gradually come into people's vision and developed rapidly. The structure reanalysis algorithm introduced in this paper is an accelerated calculation method. The core idea of this algorithm is to avoid the complete analytical calculations after the structure modification, and reduce the calculation scale, save the calculation time, improve the efficiency of CAE simulation effectively on the premise of meeting the requirements of structure accuracy. The objective of this paper that is based on the initial third-order modal information of the truck structure is to control the overall quality of the structure. And it has important guiding significance for practical production. In this paper, different design variables are set in combination with the structural reanalysis algorithm. While the parameters of design variables are modified, sensitivity information analysis and Taylor expansion theorem are used to verify the feasibility and accuracy of the structural reanalysis method in optimal calculation
\end{abstract}

\section{Introduction}

\subsection{Structure reanalysis algorithm}

Structure reanalysis algorithm is a kind of accelerating algorithm, which can quickly and effectively solve the calculation problem through the original structure information obtained from the previous complete analysis and solution of the structure. With the rapid development of computer science and technology corresponding to algorithms, the computational efficiency of large-scale structures is also improving, and the requirement of structural reanalysis is higher and higher in practice. Therefore, the application of reanalysis algorithm is becoming more and more extensive.

As early as the 1960s, the study of reanalysis problem had begun. After the painstaking research and development of scholars from generation to generation, the structural reanalysis algorithm has been relatively complete up to now, and the fields involved in the algorithm have also developed from simple linear problems to nonlinear and structural dynamic reanalysis.

Kirsch developed the combinatorial approximation method $^{[1,2]}$.The CA method combines the high precision of the direct method with the high efficiency of the local approximation method. On the basis of CA method, Chen et al. proposed the iterative combination approximation method (ICA) ${ }^{[3]}$. Wu et al. also made some contributions to the CA approach. In addition to the CA algorithm ${ }^{[4-6]}$, Levy et al. used the modified initial design to calculate the inverse matrix of the modified stiffness matrix ${ }^{[7]}$.Wu et al. expanded the application scope of this method ${ }^{[8]}$ and made it reasonably applicable to structural changes ${ }^{[9]}$ and degrees of freedom changes.

In this paper, the reanalysis algorithm is combined with the model, and the model sensitivity parameters are derived and calculated in detail with an example of a certain model truck as the main research object. Within a certain range of intensive reading, it aims to reduce the calculation workload and improve the work efficiency, so as to have a certain guiding role for the analysis of working conditions under real conditions.

\subsection{Sensitivity analysis}

Sensitivity analysis is the derivative of objective function and constraint function with respect to design variables is solved for structural optimization problems. The significance of sensitivity analysis lies in guiding structural modification. In addition, the mathematical models of structural optimization problems are nonlinear optimization models, and efficient solution methods require solving the derivative of the response with respect to the design variable, so as to construct the iterative scheme. Each iteration of structural optimization requires sensitivity information, and the full use of sensitivity information can greatly reduce the design time.

\subsection{Difference method}

The derivative of displacement vector $\mathbf{X}$ with respect to the $i$ th design variable $x_{i}$ can be approximately calculated by the difference method:

$$
\frac{\partial \mathbf{X}}{\partial x_{i}} \approx \frac{\mathbf{X}\left(x_{i}+\Delta x_{i}\right)-\mathbf{X}\left(x_{i}\right)}{\Delta x_{i}}
$$

ggk@jlu.edu.cn 
The step size $\Delta x_{i}$ is a small disturbance near the design variable. The smaller this value is, the higher the accuracy of the difference method will be. When the sensitivity of $\mathrm{n}$ design variables is separately obtained, the formula must be solved $\mathrm{n}$ times. $\mathrm{n}+1$ trigonometric decompositions and back-substitute calculations are respectively required. The difference method has the advantages of simple concept and easy programming in solving structural sensitivity. But its disadvantages are large calculation amount and unstable precision.

\section{Methods}

\subsection{Difference method}

The step When the design variable is $\mathrm{X}$, the characteristic equation of the structure is:

$$
\mathbf{K}(x) \varphi_{i}(x)=\lambda_{i}(x) \mathbf{M}(x) \varphi_{i}(x), i=1,2, \ldots \ldots, n
$$

Where $\lambda_{i}(x), \varphi_{i}(x), i=1,2, \ldots \ldots, n$ is the ith eigenpair of the structure, and the eigenvector satisfies: $\varphi_{i}^{T}(x) \mathbf{M}(x) \varphi_{j}(x)=\delta_{i j}, i=1,2, \ldots \ldots, n ; j=1,2, \ldots \ldots, n$

When $i=1,2, \ldots \ldots, n$, there are:

$\frac{\partial \mathbf{K}(x)}{\partial x} \varphi_{i}(x)+\mathbf{K}(x) \frac{\partial \varphi_{i}(x)}{\partial x}=\frac{\partial \lambda_{i}(x)}{\partial x} \mathbf{M}(x) \varphi_{i}(x)+\lambda_{i}(x) \frac{\partial \mathbf{M}(x)}{\partial x} \varphi_{i}(x)$

$+\lambda_{i}(x) \frac{\partial \varphi_{i}(x)}{\partial x}$

$\frac{\partial \varphi_{i}^{T}(x)}{\partial x} \mathbf{M}(x) \varphi_{i}(x)+\varphi_{i}^{T}(x) \frac{\partial \mathbf{M}(x)}{\partial x} \varphi_{i}(x)+\varphi_{i}^{T}(x) \mathbf{M}(x) \frac{\partial \varphi_{i}(x)}{\partial x}=0$

Arrange the above two formulas, and get:

$$
\begin{gathered}
{\left[\mathbf{K}(x)-\lambda_{i}(x) \mathbf{M}(x)\right] \frac{\partial \varphi_{i}(x)}{\partial x}-\mathbf{M}(x) \varphi_{i}(x) \frac{\partial \lambda_{i}(x)}{\partial x}=\left[\lambda_{i}(x) \frac{\partial \mathbf{M}(x)}{\partial x}-\frac{\partial \mathbf{K}(x)}{\partial x}\right] \varphi_{i}(x)} \\
2 \varphi_{i}^{T}(x) \mathbf{M}(x) \frac{\partial \varphi_{i}(x)}{\partial x}=-\varphi_{i}^{T}(x) \frac{\partial \mathbf{M}(x)}{\partial x} \varphi_{i}(x)
\end{gathered}
$$

Write it in matrix form:

$$
\left[\begin{array}{cc}
\mathbf{K}(x)-\lambda_{i}(x) \mathbf{M}(x) & -\mathbf{M}(x) \varphi_{i}(x) \\
2 \varphi_{i}^{T}(x) \mathbf{M}(x) & 0
\end{array}\right]\left[\begin{array}{c}
\frac{\partial \varphi_{i}(x)}{\partial x} \\
\frac{\partial \lambda_{i}(x)}{\partial x}
\end{array}\right]=\left[\begin{array}{c}
\lambda_{i}(x) \frac{\partial \mathbf{M}(x)}{\partial x}-\frac{\partial \mathbf{K}(x)}{\partial x} \\
-\varphi_{i}^{T}(x) \frac{\partial \mathbf{M}(x)}{\partial x}
\end{array}\right]^{T}\left[\varphi_{i}(x)\right]
$$

$$
\text { By calculating and replacing } \frac{\partial \varphi_{i}(x)}{\partial x} \text { in equation }
$$

A, we can get:

$$
\frac{\partial \lambda_{i}(x)}{\partial x}=\frac{\varphi_{i}^{T}(x)\left[\frac{\partial \mathbf{K}(x)}{\partial x}-\lambda_{i}(x) \frac{\partial \mathbf{M}(x)}{\partial x}\right] \varphi_{i}(x)}{\varphi_{i}^{T}(x) \mathbf{M}(x) \varphi_{i}(x)}
$$

Among them, $\frac{\partial \mathbf{K}(x)}{\partial x}$ and $\frac{\partial \mathbf{M}(x)}{\partial x}$ can be obtained by analytic method or difference method.

\subsection{Sensitivity analysis and Taylor expansion}

Sensitivity analysis of a structure is the key to estimating the relationship between the possible responses of a structure with different design variables of the structure.
In this paper, sensitivity analysis and Taylor's expansion theorem are used to approximate the expressions between the responses and the modifications of structural design variables. The assumption is made as follows: the structural design variable is $x_{i}, i=1,2, \ldots \ldots, m$; the initial mass and stiffness matrix of the structure is expressed as $\mathbf{M}_{0}$ and $\mathbf{K}_{0}$; after minor modification of the structure, the modified mass and stiffness matrix is denoted as

$$
\begin{aligned}
& \mathbf{K}=\mathbf{K}_{0}+\sum_{i=1}^{m} \Delta \mathbf{K}\left(x_{i}\right) \\
& \mathbf{M}=\mathbf{M}_{0}+\sum_{i=1}^{m} \Delta \mathbf{M}\left(x_{i}\right)
\end{aligned}
$$

In the above equation, $\Delta \mathbf{K}\left(x_{i}\right)$ and

$\Delta \mathbf{M}\left(x_{i}\right)$ are functions of $x_{i}, i=1,2, \ldots \ldots, m$.Considering the stiffness and mass matrix and the design variable modification, the response relationship is shown as follows:

$$
\begin{aligned}
& \mathbf{K}=\mathbf{K}_{0}+\sum_{i=1}^{m} \frac{\partial \mathbf{K}_{0}}{\partial x_{i}} \Delta x_{i} \\
& \mathbf{M}=\mathbf{M}_{0}+\sum_{i=1}^{m} \frac{\partial \mathbf{M}_{0}}{\partial x_{i}} \Delta x_{i}
\end{aligned}
$$

For micro modification of the structure, it is assumed that the modification of the initial design variable of the structural system is $\Delta x_{i}, i=1,2, \ldots \ldots, m$ and the corresponding eigenvalue is $\lambda_{j}\left(\Delta x_{1}, \Delta x_{2}, \ldots \ldots, \Delta x_{m}\right)$, and then a target value of the eigenvalue is set as $\lambda$. In order to obtain the target value set by us, the following formula needs to be constructed and the minimum absolute value of its difference value is taken, as shown below:

$$
\min \left[\lambda-\lambda_{j}\left(\Delta x_{1}, \Delta x_{2}, \ldots \ldots, \Delta x_{m}\right)\right]^{2}
$$

In this study, first-order Taylor expansion theorem is introduced and applied to the eigenvalue $\lambda_{j}\left(\Delta x_{1}, \Delta x_{2}, \ldots \ldots, \Delta x_{m}\right)$, as follows:

$$
\lambda_{j}\left(\Delta x_{1}, \Delta x_{2}, \ldots \ldots, \Delta x_{m}\right)=\lambda_{j 0}+\sum_{i=1}^{m} \frac{\partial \lambda_{j}}{\partial x_{i}} \Delta x_{i}
$$

Then substitute the above equation and use the least square method to calculate, as follows:

$$
\lambda-\lambda_{j 0}=\sum_{i=1}^{m} \frac{\partial \lambda_{j}}{\partial x_{i}} \Delta x_{i}
$$

It is not difficult to see from the above formula that if you want to obtain the value of a Certain Taylor expansion (including the first and second order), you need to calculate the first (or second order) partial derivative value corresponding to the eigenvalue at the point 
$x_{i}, i=1,2, \ldots \ldots, m$, and the calculation method is the central difference method. If any binary function $f(x, y)$ is taken and the differential variable is set, the difference fraction shown below can be obtained:

$$
\begin{aligned}
\frac{\partial f}{\partial x} & =\frac{f(x+t, y)-f(x-t, y)}{2 t} \\
\frac{\partial f}{\partial y} & =\frac{f(x, y+t)-f(x, y-t)}{2 t} \\
\frac{\partial^{2} f}{\partial x^{2}} & =\frac{f(x+t, y)-f(x-t, y)-2 f(x, y)}{t^{2}} \\
\frac{\partial^{2} f}{\partial y^{2}} & =\frac{f(x, y+t)-f(x, y-t)-2 f(x, y)}{t^{2}} \\
\frac{\partial^{2} f}{\partial x \partial y} & =\frac{f(x+t, y-t)-f(x-t, y+t)}{4 t^{2}} \\
& -\frac{f(x+t, y+t)-f(x-t, y-t)}{4 t^{2}}
\end{aligned}
$$

From the above formula, it can be seen that the sensitivity information of the structure is expanded by Taylor (taking the first and second order), and the approximate expression relationship between the defined design variable modification and the structural response can be obtained, and then the optimization algorithm (such as the least square method) can be used to solve the optimal solution of the target in the feasible region of the structure.

\section{Calculations}

Large finite element truck body model example is presented.

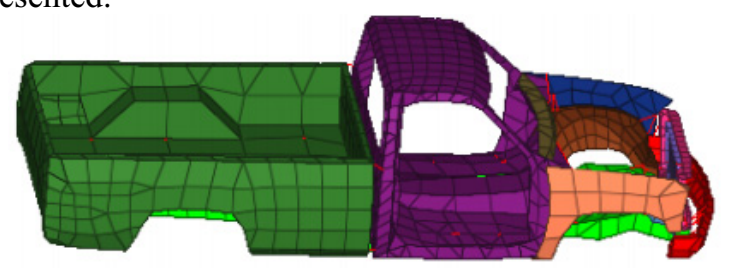

Figure 1: The truck model

Independent variable: The chassis Rails and Bed of the body are selected as independent variables, as shown in Figure 4.1 and Figure 4.2. Let the guide plate thickness be $\mathrm{X}$ and the thickness of the carriage be $\mathrm{Y}$, and take the step length $\mathrm{t}=0.01$.

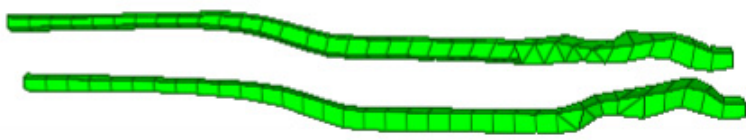

Figure 2: The chassis rail of a car body

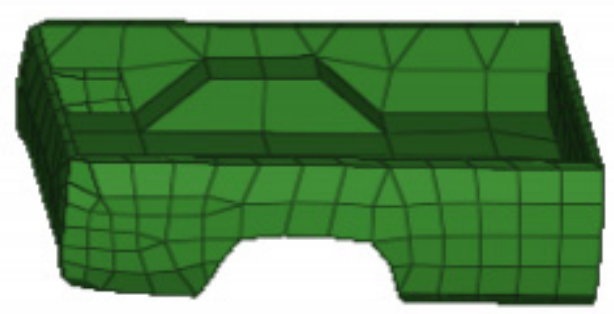

Figure 3: The bed of the body

Constraints: ensure that the system's modes and stiffness are not reduced. The mode of the system is expressed by the natural frequency, and the stiffness of the system is measured by the simulated deformation and displacement under loading conditions. Since the finite element software USES approximate solution to calculate the modal, the accuracy of this example is relatively high, so the natural frequency is calculated from the eigenvalue of the augmented matrix composed of the mass matrix of the stiffness matrix.

Objective: to reduce model quality.

According to the difference method presented in this paper, the thickness of independent variables is modified in turn according to the step length to obtain the maximum displacement point and the modified mass of each modified model. Then, the first and second derivatives of displacement and mass with respect to independent variables $\mathrm{X}$ and $\mathrm{Y}$ are calculated according to the difference method as illustrated in the table below:

Table1. First derivative of natural frequency

\begin{tabular}{ccc}
\hline & $\frac{\partial}{\partial x}$ & $\frac{\partial}{\partial y}$ \\
\hline$\partial S$ & -0.071 & -4.874 \\
$\partial M$ & 0.025 & 0.12 \\
\hline
\end{tabular}

Table 2 Second partial derivatives

\begin{tabular}{cccc}
\hline & $\frac{\partial}{\partial x^{2}}$ & $\frac{\partial}{\partial y^{2}}$ & $\frac{\partial}{\partial x \partial y}$ \\
\hline$\partial^{2} S$ & 0 & 6.2 & -0.025 \\
$\partial^{2} M$ & -1 & 0 & 0.25 \\
\hline
\end{tabular}

The first and second Order Taylor expansion expressions of displacement $\mathrm{S}$ are as follows:

$S_{1}=-5.14801+0.071(\mathrm{x}-4)+4.874(\mathrm{y}-3)$

$S_{2}=-5.14801+0.071(\mathrm{x}-4)+4.874(\mathrm{y}-3)-6.2(\mathrm{y}-3)^{2}+0.025(\mathrm{x}-4)(\mathrm{y}-3)$

The first and second order Taylor expansion expressions of mass $M$ are as follows:

$M_{1}=0.4577+0.025(\mathrm{x}-4)+0.12(\mathrm{y}-3)$

$M_{2}=0.4577+0.025(\mathrm{x}-4)+0.12(\mathrm{y}-3)-(\mathrm{x}-4)^{2}+0.25(\mathrm{x}-4)(\mathrm{y}-3)$

In

this

$\lambda_{1}=538.36, \lambda_{2}=2308.8, \lambda_{3}=2984.7$

The first three natural frequencies can be calculated: $\omega_{1}=3.695, \omega_{2}=7.651, \omega_{3}=8.709$ 
After calculating the natural frequencies of all the modified models, the first and second derivatives of the first three natural frequencies can be obtained according to the difference method:

Table3. First derivative of natural frequency

\begin{tabular}{cccc}
\hline & $\partial \omega_{1}$ & $\partial \omega_{2}$ & $\partial \omega_{3}$ \\
\hline$\frac{\partial}{\partial x}$ & 0.026 & 0.011 & 0.482 \\
$\frac{\partial}{\partial y}$ & & & \\
\hline
\end{tabular}

Table4. Second derivative of natural frequency

\begin{tabular}{cccc}
\hline & $\partial^{2} \omega_{1}$ & $\partial^{2} \omega_{2}$ & $\partial^{2} \omega_{3}$ \\
\hline$\frac{\partial}{\partial x^{2}}$ & -0.0075 & -0.072 & -5.196 \\
$\frac{\partial}{\partial y^{2}}$ & -0.0014 & 14.514 & 25.704 \\
$\frac{\partial}{\partial x \partial y}$ & 0.0004 & -0.0205 & 0.1896 \\
\hline
\end{tabular}

Then the first and second Order Taylor expansion expressions of the first natural frequency $\omega_{1}$ are:

$$
\begin{aligned}
\omega_{1} & =3.6944+0.026(\mathrm{x}-4)+0.0007(\mathrm{y}-3) \\
\omega_{1} & =3.6944+0.026(\mathrm{x}-4)+0.0007(\mathrm{y}-3)-0.0075(\mathrm{x}-4)^{2}-0.0014(\mathrm{y}-3)^{2} \\
& +0.0004(\mathrm{x}-4)(\mathrm{y}-3)
\end{aligned}
$$

Then the first and second Order Taylor expansion expressions of the second natural frequency $\omega_{2}$ are:

$$
\begin{aligned}
\omega_{2} & =7.6313+0.011(\mathrm{x}-4)+1.19125(\mathrm{y}-3) \\
\omega_{2} & =7.6313+0.011(\mathrm{x}-4)+1.19125(\mathrm{y}-3)-0.072(\mathrm{x}-4)^{2}+14.514(\mathrm{y}-3)^{2} \\
& -0.0205(\mathrm{x}-4)(\mathrm{y}-3)
\end{aligned}
$$

Then the first and second Order Taylor expansion expressions of the third natural frequency $\omega_{3}$ are:

$\omega_{3}=8.6984+0.482(\mathrm{x}-4)-0.4486(\mathrm{y}-3)$

$\omega_{3}=8.6984+0.482(\mathrm{x}-4)-0.4486(\mathrm{y}-3)-5.1956(\mathrm{x}-4)^{2}+25.7044(\mathrm{y}-3)^{2}$ $+0.1896(\mathrm{x}-4)(\mathrm{y}-3)$

\section{Conclusion}

Compared with the finite element software optimization, the CA modal re-analysis algorithm studied in this paper saves the time of solution and improves the efficiency of calculation. It has great potential in the current trend of increasingly com-plicated models. Times are advancing and computer technology is developing faster and faster. As an auxiliary means of acceleration, the reanalysis method will be more and more closely combined with software. It will surely better meets the requirements of future industrial computing development.

\section{Acknowledgement}

This work was supported by the National Science Foundation of China (Grant No. 11502092), the Plan for Education Development of Jilin Province (Grant No. JJKH20190142KJ) and the Plan for Scientific and Technological Development of Jilin Province (Grant No. 20200201272JC).

\section{References}

1. Kirsch U, PAPALAMBROS PY. Structural reanalysis for topological modifications - a unified approach [J]. Struct Multidiscip Optim, 2001, 21(5): 333-44.

2. Kirsch. U., Papalambros. P. Y. Exact and accurate solutions in the approximate reanalysis of structures [J]. Aiaa Journal, 2001, 39(11): 2198-205.

3. Chen.S.H, Yang.Z.J. A universal method for structural static reanalysis of topological modifications [J]. International Journal for Numerical Methods in Engineering, 2004, 61(5): 673-86.

4. Liu.H.F.,Wu.B.S, Li.Z.G. Simple iteration method for structural static reanalysis $[\mathrm{M}] .2015$, 141(2): 04014111

5. Wu.B,Li.Z. Static reanalysis of structures with added degrees of freedom [J]. Communications in Numerical Methods in Engineering, 2005, 22(4): 26981.

6. Wu.B.S, Lim.C.W, Li.Z.G. A finite element algorithm for reanalysis of structures with added degrees of freedom [J]. Finite Elements in Analysis and Design, 2004, 40(13-14): 1791-801.

7. Levy. R., Kirsch. U., Liu. S. Reanalysis of trusses using modified initial designs [M]. Springer-Verlag New York, Inc., 2000.

8. Wu.B, Li.Z, Li.S. The implementation of a vectorvalued rational approximate method in structural reanalysis problems $[\mathrm{J}]$. Computer Methods in Applied Mechanics and Engineering, 2003, 192(1314): $1773-84$.

9. Liu.H.F, Wu.B.S, Li.Z.G. Preconditioned Conjugate Gradient Method for Static Reanalysis with Modifications of Supports [J]. Journal of Engineering Mechanics, 2015, 141(2). 